\title{
Las competencias profesionales y sus tendencias fundamentales
}

DOI: https://doi.org/10.33262/ap.v2i1.23

\begin{abstract}
(c) (1) (2)(2)
BY NC SA

Professional competencies and their fundamental trends

Elizabet Prado Chaviano., ${ }^{1}$ Margarita González González., ${ }^{2}$ \& Bisleivys Jiménez Valero., ${ }^{3}$
\end{abstract}

\begin{abstract}
.
The competences have become currently one of the most controversial and interesting issues at the international level, their training in the educational context, has brought about changes in the teaching-learning process of higher education where four trends are manifested in its conception. This research aims to present theoretical foundations that refer to trends in professional skills with a critical analysis of them as well as the definition of professional skills by the authors.
\end{abstract}

Keywords: competencies, professionals, trends, modes of action, psychological configuration, capabilities.

\section{Resumen.}

Las competencias se han convertido en la actualidad uno de los temas más polémicos y de interés a nivel internacional, su formación en el contexto educacional, ha traído aparejados cambios en el proceso de enseñanza aprendizaje de la educación superior donde se manifiestan cuatro tendencias en su concepción. Esta investigación tiene como objetivo presentar fundamentos teóricos que refieren las tendencias de las competencias profesionales

\footnotetext{
${ }^{1}$ Universidad de Matanzas. La Habana, Cuba. elizabetpradochaviano7979@gmail.com

${ }^{2}$ Universidad de Matanzas. La Habana, Cuba. margarita.gonzalez@umcc.cu

${ }^{3}$ Universidad de Matanzas. La Habana, Cuba. bisleivys.jimenez@umcc.cu
} 
con un análisis crítico de las mismas, así como la definición de las competencias profesionales por los autores.

Palabras claves: competencias, profesionales, tendencias, modos de actuación, configuración psicológica, capacidades.

\section{Introducción.}

Uno de los retos más significativos que hoy día se plantea la Educación Superior Contemporánea, es la formación de profesionales competentes que sean verdaderos transformadores de su entorno y comprometidos con el desarrollo social (Aupetit, 2014), de acuerdo a los constantes cambios que se originan en los escenarios ocupacionales y educativos (Prado, Baujín y Alonso, 2017 a y b).

En el contexto universitario actual, esta formación de profesionales se realiza de modo consciente y sobre bases científicas, para garantizar la preparación integral de los estudiantes universitarios, con el fin de lograr profesionales revolucionarios, cultos, competentes, independientes y creadores, para que puedan desempeñarse exitosamente en los diversos sectores de la economía y de la sociedad en general, Ministerio de Educación Superior (MES, 2018b) y en concordancia con lo declarado por la Junta de Acreditación Nacional (JAN), que asume el sistema de evaluación y acreditación de carreras universitarias (SEA-CU), donde se plantea la necesidad de lograr en las universidades cubanas: "El egreso de profesionales con elevado nivel de calidad, independientemente de la modalidad de estudio de las carreras universitarias, con un desempeño ético, competente y transformador en la solución de problemas territoriales" (MES, 2018a, p.5).

En los estudios analizados sobre la formación de competencias profesionales en el entorno educativo, se destacan autores como (Pérez, 2014; Rey, 2014; Pérez y González, 2015; Fonseca, 2017; Guzmán, 2017; Nkola, 2017; Ramos, 2017 y Pérez, 2019) que realizan investigaciones desde las asignaturas y las carreras, en las cuales se evidencia el perfeccionamiento del concepto de competencia profesionales en cuanto a su rigor, profundidad y amplitud, donde no solo están presentes los conocimientos y habilidades en el ejercicio de una profesión, sino también se refiere a los modos de actuación en el entorno socioeconómico en el que desempeña su función profesional.

Estas investigaciones se encuadran en cuatro tendencias fundamentales, como: los modos de actuación donde se enfatiza en el desempeño laboral (Mohamadi y Malekshahi, 2018; Peltran, Niculuta y Petcu, 2018 y Moghabghab, Tong, Hallaran y Anderson, 2018), las configuraciones sicológicas complejas que integran elementos cognitivos, metacognitivos, axiológicos, personológicos y motivacionales (Boritz y Carnaghan, 2017; Sánchez, Soler, Martín, López, Ageno, Cabré y Gibert, 2018; Asonitou y Hassall, 2019; Bogoviz, Gulyaeva, Semenova y Lobova, 2019 y Salnikova, Salnikova, Soroka y Stolyarova, 2020); las capacidades como la integración de conocimientos habilidades y valores (Fuentes, 2002 y 
Corral, 2004) y desde la integración, la cual constituye la tendencia a la que se adscribe la investigadora, que combina la formación de conocimientos, habilidades, valores, las motivaciones y el desempeño profesional (Capó, Castillo y González, 2013; Santos, 2016; Guzmán, 2017 y Martínez, Álvarez de Eulate y Villardón, 2017).

Esta investigación tiene como objetivo: presentar fundamentos teóricos que refieren las tendencias de las competencias y un análisis crítico de las mismas.

Para lo cual se apoya en métodos como el analítico-sintético que se pone de manifiesto en el procesamiento de la información que ofrecen las fuentes de información y llegar a conclusiones, a fin de determinar los diferentes tendencias y los criterios relacionados con las competencias profesionales, lo cual se manifiesta a partir de precisiones enunciadas por los autores en términos de coincidencias, divergencias, aportes y limitaciones de los elementos abordados y el inductivo-deductivo que permitió el razonamiento de los elementos generales y particulares de las competencias profesionales.

\section{Desarrollo.}

El término competencia no tiene mucha antigüedad en las Ciencias Pedagógicas y sobre el inicio de su utilización hay diferentes criterios; para algunos se extiende desde finales de la década del 50 en los Estados Unidos, a partir de los fundamentos teóricos que sustentaba el cognitivismo (Castellanos, Llivina y Fernández, 2003). Son reconocidos otros estudiosos del tema que aseguran que el término se acuñó en el 1957 vinculado a las artes, cuando se definió como capacidades y disposiciones para la interpretación y la actuación (Aguirre, 2005). En ese mismo año, Chomsky asume el término competencia como un concepto clave en su teoría, para explicar lo que era considerado como la habilidad humana de aprender la primera lengua, cualquiera que esta sea. A criterio de la autora, su acercamiento al concepto de competencia se vio limitado por la separación que hizo entre los conceptos de competencia y actuación, al no reconocer la mutua correspondencia y el condicionamiento bidireccional existente entre ambos procesos, e imprimiéndole al término competencia un concepto puramente mentalista, innatista, posición teórica superada por él años más tarde.

Otros estudios han privilegiado diferentes aspectos en el análisis de las competencias, ya sea por el desempeño laboral, (McClelland, 1973 y Mertens, 2000), los cuales consideran que el estudio de las competencias comienza con una búsqueda orientada al hallazgo de criterios científicos explicativos de la eficiencia de las personas vinculado al mundo del trabajo y Mulder (2007) cuando refiere que la competencia tiene sólo dos significados esenciales, que es autoridad (o sea, ostentar la responsabilidad, la autoridad o el derecho a decidir, producir, prestar servicios, actuar, ejercer o reclamar) y capacidad (es decir, poseer los conocimientos, las aptitudes y la experiencia para ejercer) interpretación esta que a criterio de los autores, va solo al aspecto cognitivo. 
Para Norton (2017), las competencias son aquellos comportamientos observables y habituales que posibilitan el éxito de una persona en su actividad o función, mientras la Real Academia Española (RAE) en el año 2018, define la competencia como la pericia, aptitud e idoneidad para hacer algo o intervenir en un asunto determinado. A criterio de los autores esta definición es poco abarcadora, solo se refiere a aspectos cognitivos y no tiene en cuenta otros aspectos como el procedimental y afectivos que son importantes para un desempeño eficiente.

Por su parte Hernández y Fajardo (2011); González y Ramírez (2011); Huerta, Pérez y Castellano (2013); Pérez (2014); Pérez y González (2015) y Martínez, Álvarez y Villardón (2017) realizan investigaciones sobre las competencias, en las cuales reconocen dentro de las competencias, las profesionales como un factor fundamental a tratar en la educación superior y consideran su formación mediante la integración de conocimientos, habilidades, actitudes, valores motivos, aptitudes, procedimientos y destrezas, necesarias para garantizar un adecuado desarrollo profesional; las que son adquiridas a través de la experiencia, las mismas permitirán al individuo colaborar en el entorno profesional y en la entidad del trabajo en contextos singulares.

Otros autores como Pavié (2011), Ferreira y Lima (2013), Aguilar (2015) y Vélez, Delgado y Sánchez (2018) consideran las competencias profesionales como una combinación dinámica de atributos con respecto al conocimiento y su aplicación, a las actitudes y a las responsabilidades que describen los resultados del aprendizaje de un determinado programa, o cómo los estudiantes serán capaces de desenvolverse al finalizar el proceso educativo.

Por su parte, Delgadillo (2015); Ortiz, Vicedo, González y Recino (2015); Véliz, Jorna y Berra, (2016) y Ortiz (2018), refieren que las competencias profesionales se articulan en la docencia universitaria mediante el proceso de enseñanza aprendizaje para dar solución de las demandas de la profesión (Ortiz, 2003; Pavié, 2011; Pavié, 2016; Santos, 2016; Guzmán, 2017 y Pérez, 2019), criterio con el cual los autores concuerdan y agregan que, se debe incorporar, el establecimiento de los vínculos con las entidades empleadoras en función de la formación de conocimientos, habilidades, desempeños, motivos y valores que permiten desarrollar correctamente la actividad laboral sobre la base de acciones razonadas y sistemáticas que viabilizan la contribución profesional.

Estas competencias profesionales, tienen múltiples clasificaciones, dentro de las cuales se encuentran las competencias genéricas comunes a la mayoría de las profesiones relacionadas con las aptitudes, rasgos de la personalidad, conocimientos y valores adquiridos que se requieren en diferentes áreas ocupacionales y las competencias específicas que son las analizadas en función de esta investigación, estas competencias son la base particular del ejercicio profesional y están vinculadas a condiciones específicas de ejecución (Tejeda y Sánchez, 2010; Hernández y Fajardo 2011; Huerta, Pérez y Castellano, 2013; Zoia, Barbieri; Cortelezzi y Marseguerra, 2018 y Pérez, 2019). 
En relación a las competencias profesionales específicas los autores coinciden con (Tejeda y Sánchez, 2010, p. 29) cuando expresan: "la identidad de la actuación del profesional y se corresponden con las características de la profesión, rama o sector socio laboral, son identificadas por los gestores del proceso formativo y se socializan con los profesionales en ejercicio, relacionados con la carrera o programa universitario. Estas competencias distinguen a un profesional de otro y connotan su desempeño en los diversos contextos. Este criterio pone de manifiesto la formación de un profesional más comprometido, flexible y trascendente, que reúna valores éticos, capacidad de análisis y argumentación, que posea los conocimientos requeridos para asumir desempeños profesionales.

Los estudios realizados de estas competencias profesionales se enmarcan en cuatro tendencias principales. En la primera tendencia, estas competencias son concebidas desde los modos de actuación autores como: McClelland (1973); Nilsson, Engströmd, Florinf, Gardulf y Carlsson (2018); Mohamadi y Malekshahi (2018) y Peltran, et al (2018), conceptualizan las competencias profesionales orientadas a la eficiencia de las personas en el desempeño laboral en una situación de trabajo, con patrones que permiten comprobar si un trabajador es competente.

A criterio de los autores, esta tendencia carece de un análisis complejo, sistémico e integrador para la formación de competencias profesionales, porque no establece una vinculación en la formación profesional entre las instituciones educativas y el entorno laboral, para lo cual se integran los conocimientos, habilidades, comportamientos, valores, motivación y desempeños.

La segunda tendencia considera las competencias profesionales como configuración sicológica compleja. Autores como Castellanos et al., (2003); Helm (2015); Asonitou y Hassall (2019); Bogoviz, et al., (2019) y Salnikova, et al., (2020), expresan que las competencias profesionales conciben formaciones motivacionales, cognitivas, metacognitivas, recursos personológicos, calidad en la actuación profesional del sujeto en una actividad específica o en un contexto histórico (Castellanos, et al., 2003 y Pérez, 2019).

Los autores consideran que esta tendencia, si bien aporta un sustento teórico en términos de formación de competencias profesionales, al analizar cualidades esenciales de la personalidad, se centra en los procesos mentales del individuo y no enfatiza en el desempeño profesional.

La tercera tendencia concibe las competencias profesionales como capacidades. En este sentido, Corral (2004) y Manzi (2018), plantean que estas competencias son capacidades que se manifiestan en las actuaciones eficientes y eficaces sobre algún aspecto de la realidad personal, social, natural o simbólica, a partir de los conocimientos habilidades y valores que se adquieren en el proceso de enseñanza aprendizaje. Para los autores, esta tendencia es 
limitada porque se excluyen aspectos importantes tales como la motivación, los comportamientos, las limitaciones y logros en el aprendizaje y los desempeños.

La cuarta tendencia, a la que los autores se adscriben, refiere las competencias profesionales desde la integración al combinar los conocimientos, habilidades y actitudes del sujeto, coordinadas e integradas en la acción, desempeño profesional, resolución de problemas profesionales, idoneidad, evaluación, anticipación y decisión; comportamientos, facultad de análisis, toma de decisiones, asociados a saber, saber hacer, saber ser, saber poder, querer o disposición para saber (Capó, Castillo y González, 2013 y Martínez, et al., 2017).

Esta tendencia es tratada en universidades europeas y de América Latina (Pavié, 2011 y Aguilar, 2015), sin embargo, en la Educación Superior en Cuba, las carreras universitarias continúan en la actualidad enfatizando en el desarrollo de las habilidades profesionales y el estudio de la formación por competencia se encuentra en un estado inicial, con propuestas focalizadas en contextos muy particulares Pérez (2019) y modelos de formación por competencias profesionales (Hernández y Fajardo, 2011; Mena, 2014 y Acosta, Costales y Rosales, 2016) las cuales están en concordancia con el artículo 1 de la resolución 2/2018 donde se hace referencia a formar profesionales revolucionarios, cultos, competentes, independientes y creadores, para que puedan desempeñarse exitosamente en los diversos sectores de la economía y de la sociedad en general (MES, 2018a).

A criterio de los autores, para la formación por competencias profesionales se hace necesario definir varias etapas para lograr una adecuada formación de las mismas, lo cual se corresponde con la concepción del documento base para la elaboración del Plan de Estudio $\mathrm{E}$, donde se propone un sistema de formación continua basado en tres etapas (MES, 2015) las cuales son:

- Formación de pregrado en carreras de perfil amplio, donde la universidad tendrá mayor responsabilidad y debe: asegurar una profunda formación en los aspectos básicos y básicos específicos de cada profesión, y desarrollar en el egresado modos de actuación que les permita dar respuesta a los problemas en el eslabón de base de la profesión.

- Preparación para el empleo, donde los organismos empleadores tendrán una mayor responsabilidad: concebida y ejecutada en las entidades laborales, con el propósito de desarrollar en el recién egresado los modos de actuación profesional específicos relacionados con su puesto de trabajo.

- Formación de posgrado, donde existirá una responsabilidad compartida entre las universidades y los organismos empleadores: mediante la participación de los profesionales en programas de especialización, reorientación y actualización permanente (cursos, diplomados, especialidades de posgrado, maestrías y doctorados). 
De acuerdo con estas etapas se proyectaron tres niveles (MES, 2015) que se presentan a continuación:

- Primer nivel: con salida intermedia al concluir segundo año. Objetivo: dotar a los estudiantes de los conocimientos básicos necesarios para resolver problemas en el eslabón de base, considerando la naturaleza de estos problemas en el contexto actual inmediato.

- Segundo nivel: con salida al concluir cuarto año. Objetivo: consolidar los conocimientos adquiridos en el primer nivel a través de la solución de problemas concretos, y ampliar la formación teórica y práctica básica en el perfil profesional, orientándose a las necesidades del país a mediano plazo.

- Tercer nivel: enseñanza posgraduada. Objetivo: consolidar los conocimientos adquiridos en el pregrado y ampliar la formación teórica y práctica, considerando los avances de la ciencia y su aplicación en el contexto nacional.

A criterio de los autores la formación de las competencias profesionales en la etapa formativa tiene tres estadios: la formación inicial que se corresponde con el primer nivel y que ofrece a los estudiantes los conocimientos básicos que permiten dar solución a problemáticas de cada profesión y se desarrollan en el estudiante los modos de actuación profesional; la formación intermedia que se corresponde con el segundo nivel, donde se consolidan los conocimientos ya adquiridos y se profundiza en la formación teórica y práctica en relación a su perfil profesional y la formación avanzada, que para los autores en relación a la formación de competencias profesionales, coincide con la etapa de adiestramiento, donde los organismos empleadores tendrán una mayor responsabilidad con el propósito de desarrollar en el recién egresado los modos de actuación profesional específicos relacionados con su puesto de trabajo.

Después del análisis realizado de los conceptos y tendencias expuestos en la presente investigación, se define por los autores la formación de la competencia profesional como: un proceso continuo, complejo y sistémico, que integra conocimientos, habilidades, valores, motivaciones y desempeños que se incorporan a la actuación del estudiante en la actividad que desarrollan con la ayuda del profesor, el tutor y de otros contextos de actuación.

\section{Conclusiones.}

- Las cuatro tendencias de las competencias profesionales reflejan el carácter evolutivo del tratamiento de las mismas en cuanto a los elementos que la conforman desde los conocimientos, habilidades, valores, motivación y desempeños tanto en el proceso de enseñanza aprendizaje como en el desempeño profesional y su integración en el entorno académico, laboral y otros contextos de actuación por lo que requiere del trabajo cooperado de profesores, tutores y los estudiantes 


\section{Referencias bibliográficas.}

Castellanos, B., Llivina M.J., y Fernández, A.M. (2003). La gestión de la actividad de la ciencia e innovación tecnológica y la competencia investigativa del profesional de la educación. Revista del Instituto Pedagógico Latinoamericano y Caribeño, 9(2), p.14. Recuperado de: http//www.revtecnologia'sld.cu.

Aguirre, D A. (2005). Reflexiones acerca de la competencia comunicativa profesional. Revista de Educación. Médica, 19(3), sp. Recuperado de: http://scielo.sld.cu/scielo.php.

Mcclelland, D. (1973). Testing for Competence Rather than for Intelligence en American Psychologist. Recuperado de: http://www.lichaoping.com/wpcontent/ap7301001.pdf.

Mena, T. A. (2014). Sistema para el trabajo metodológico en las disciplinas docentes complejas. Una estrategia para su implementación en la disciplina Contabilidad en la carrera de Contabilidad y Finanzas de la Universidad de Pinar del Río (Tesis doctoral, Universidad de Pinar del Río Hermanos Saiz Montes de Oca, Cuba).

Mertens, L. (2000). La gestión por competencia laboral en la empresa y la formación profesional. OEI. Recuperado de: http://www.oei.es.

Mulder, M. (2007). Introducción al número especial sobre competencias. Competencia: la esencia y la utilización del concepto en la formación profesional inicial y permanente. Revista Europea de Formación Profesional, 40(1), p.58-96. Recuperado de: http://www.dialnet.unirioja.es/servlet/dcfichero_articulo?codigo=2316165.

Norton, R. (2017). Alineamiento de las competencias laborales. Revista Retos de la Dirección, 11(1), sp. Recuperado de: http//scielo.sld.cu.

Ortiz, E. (2003).Competencias y valores profesionales. Revista Pedagogía Universitaria, 6(2).p.25-48. Recuperado de: http://www.revistas.mes.edu.cu.

Ortiz, M. (2018). Modelo pedagógico para el mejoramiento del desempeño docente con enfoque de competencias en la especialidad de pediatría. Revista Habanera de Ciencias Médicas, 17(1), sp. Recuperado de: http://scielo.sld.cu/scielo.php.

RAE. (2018). Definición del término competencia. Diccionario de la Real Academia Española. España.

Hernández, G.C., y Fajardo, I. (2011). Sistema de actividades para contribuir a la formación de la competencia profesional para el análisis en estudiantes de la carrera Contabilidad y Finanzas en la FUM de Colón (CD - ROM). Matanzas: Universidad de Matanzas Camilo Cienfuegos.

Huerta, J., Pérez, I. S., y Castellanos, A. R. (2013). Desarrollo curricular por competencias profesionales integrales. Recuperado de: http://educar.jalisco.gob.mx/13/13Huerta.html.

González, M., y Ramírez, I. (2011). La formación de competencias profesionales: un reto en los proyectos curriculares universitarios. Odiseo, Revista Electrónica de Pedagogía, 8(6), p.1-7 Recuperado de: http//:odiseo@odiseo.com.mx. 
Pérez, E. (2014). La formación de competencias profesionales en los estudiantes de técnico medio en informática mediante proyectos informático (Tesis doctoral, Universidad de Ciencias Pedagógicas José de La Luz y Caballero, Holguín, Cuba).

Pérez, T. (2019). La formación inicial de la competencia profesional resolver problemas económicos mediante el proceso de enseñanza aprendizaje de la Econometría (Tesis doctoral, Universidad de Matanzas Sede Juan Marinello, Matanzas, Cuba).

Pérez, T., y González, W. (2015). Desarrollo de competencias profesionales en los estudiantes de la carrera Contabilidad y Finanzas desde la enseñanza de los conocimientos de Econometría. Revista Electrónica Formación y Calidad Educativa, 3(2), p.58-62. Recuperado de: http//www.refcale.urlean.edu.ec.

Petran, H.-A., Niculuta, M.-C., y Petcu, C. (2018). Competences development - Towards an effective implementation of nZEB in Romania. Editorial Nearly Zero Energy Communities. Rumania.

Pavié, A. (2011). Formación docente: hacia una definición del concepto de e competencia profesional docente. Revista Electrónica Interuniversitaria de Formación del Profesorado 14 (1), p. 67-80. Recuperado de: http//dialnet.uniroja.es.art.

Pavié, A. (2016). Las competencias profesionales del profesorado de lengua castellana y comunicaciones en Chile: Aportaciones a la formación inicial. Recuperado de: http//uvadoc.uva.es/handle/10324/2794.

Martínez, M., Álvarez de Eulate, C. Y., y Villardón, L. (2017). Estudos e desenvolvimento das competências profissionais. Revista Iberoamericana de Educación. 74(1), p.171192. Recuperado de: http//dialnet.uniroja.es/ejemplar/461813.

Aguilar, J. (2015). Competencias específicas Tuning en programas de administración: Colombia y su región sur occidental. Revista Universidad EAFIT, 35(59), p.2336.doi:http//10.18634/ctxj,4v1i,410.

Ferreira, K. C., y Lima, P.G. (2013). Proyecto tuning América Latina en las universidades brasileras: características y ámbitos en el área de educación. Revista Paradigma 34(1), 83-96. Recuperado de: http//ve.scielo.org.

Delgadillo, J. (2015). Modelos educativos por competencia y por comprensión en la educación superior. (Tesis doctoral, Universidad de Granada, España).

Vélez, A., Delgado, L.D., y Sánchez. (2018). Análisis prospectivo de las competencias genéricas Tuning -Alfa Revista El Ágora, 18(1), p. 130-151. Recuperado de: http//dx.doi.org/10.21500/16578.31.3446.

Véliz, P.L., Jorna, A.R., y Berra, E.M. (2016). Consideraciones sobre los enfoques, definiciones y tendencias de las competencias profesionales. Revista de Educación Médica Superior, 30(2), s.p. Recuperado de: http://scielo.sld.cu/scielo.php.

Ortiz, M., Vicedo, A., González, S., y Recino, U. (2015). Las múltiples definiciones del término competencia y la aplicabilidad de su enfoque en ciencias médicas. Revista EDUMECENTRO 7(39), sp. Recuperado de: http://scielo.sld.cu/scielo.php. 
Santos, A. (2016). Modelo Espiral de Competencias Docentes TICTACTEP aplicado al Desarrollo de Competencias Digitales. Revista Educativa Hekademos 4(2), p. 39-48. Recuperado de: http//www.researchgate.net.

Guzmán, Y. (2017). Metodología para la determinación de competencias de gestión del profesor principal del año académico en las instituciones de educación superior cubanas (Tesis doctoral, Instituto Central de Ciencias Pedagógicas Enrique José Varona, La Habana, Cuba).

Helm, C. (2015). Determinants of competence development in accounting in upper secondary education. Empirical Research in Vocational Education and Training. Revista Journal Springer, 7(10), p 1-10, doi: http//10.1186/s40461-015-0022-8.

Tejeda, R., y Sánchez, P. (2010). La formación basada en competencias profesionales en los contextos universitarios. Holguín: Universidad de Holguín. Centro de Estudios sobre Ciencias de la Educación Superior, 10-29.

Nilsson, J., Engströmd, M., Florinf, J., Gardulf, A., y Carlsson, M. (2018). A short version of the nurse professional competence scale for measuring nurses' self-reported competence. Revista Nurse Education Today, 71(6), p. 223-239. doi: http: //10.1016/j.nedt.2018.09.028.

Zoia, M. G., Barbieri, L., Cortelezzi, F.,y Marseguerra, G. (2018). The determinants of Italian firms' technological competencies and capabilities. Revista Eurasian Business Review, 8(4), p. 453-476.doi: http// 10.1007/s40821-018-0103-2.

Corral, R. (2004). El currículo docente basado en competencias. Recuperado en: http//biblioteca.clacso.edu.ar.cips.

Manzi, G.E. (2018). Diseño y estructuración del currículum universitario de las carreras de publicidad, análisis de la formación de competencias (Tesis de maestría, Universidad de Ciencias Pedagógicas José de La Luz y Caballero, Holguín, Cuba).

MES. (2015). Documento base para la elaboración del Plan de Estudio E. La Habana (Cuba).

MES. (2018a). Resolución Ministerial No. 150/2018: Reglamento del Sistema de Evaluación y Acreditación de la Educación Superior. La Habana (Cuba).

Acosta, M., Costales, Z., y Rosales, B. (2016). Formación por competencias profesionales en la carrera Periodismo. Revista Cubana de Educación Superior, 35 (1), p.75-84. Recuperado de: http//www.scielo.sld.cu/scielo.php?script=sci_abstract\&pid=SO25743142016000100007.

Capó, J. R., Castillo, J. D., y González, S. (2013). Sistema de gestión integrada de capital humano para las universidades cubanas. Revista Congreso Universidad, 2(3), p.2-13. Recuperado de: http//revista.congresouniversidad.cu/index.php/rcu/article/view/511.

Mohamadi, Z., y Malekshahi, N. (2018). Designing and validating a potential formative evaluation inventory for teacher competences. Revista Language Testing in Asia, 8(1): 6, doi: http//10.1186/s40468-018-0059-2. 
Asonitou, S., y Hassall, T. (2019). Which skills and competences to develop in accountants in a country in crisis? Revista Journal of Management Education, 17(3), p. 124-132. doi: http: //10.1016/j.ijme.2019.100308.

Bogoviz, A.V., Gulyaeva, T.I., Semenova, E.I., y Lobova, S.V. (2019). Transformation changes in the system of professional competences of modern specialists in the conditions of knowledge economy's formation and the innovational approach to training. Revista Bussines, 2(8), p.193-200, doi: http//10.1007/978-3-319-94310-719.

Salnikova, M., Salnikova, Y., Soroka, M., y Stolyarova, V. (2020). Students' Independent Learning Activity as an Effective Method of Acquiring Professional Competencies. En: Proceedings of the AHFE 2019 International Conference on Human Factors in Training. Revista Education, and Learning Sciences, 25 (2), p. 391-399-28 doi: http// 101.1007/9783030-201357-39. 


\section{Para citar el artículo indexado}

Prado Chaviano, E., González González, M., \& Jiménez Valero, B. (2020). Las competencias profesionales y sus tendencias fundamentales. AlfaPublicaciones, 2(1), 44-55. https://doi.org/10.33262/ap.v2i1.23

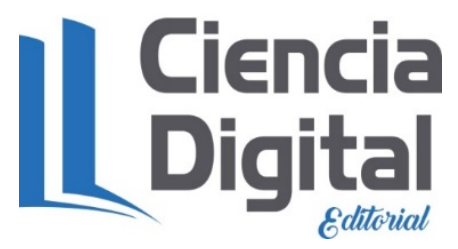

El artículo que se publica es de exclusiva responsabilidad de los autores y no necesariamente reflejan el pensamiento de la Revista Alpha Publicaciones.

El artículo queda en propiedad de la revista y, por tanto, su publicación parcial y/o total en otro medio tiene que ser autorizado por el director de la Revista Alpha Publicaciones.
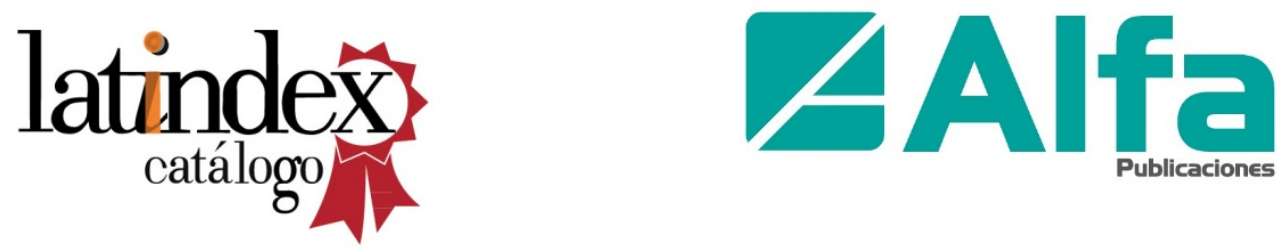